Transactions of the SDPS:

Journal of Integrated Design and Process Science

21 (3), 2017, 1-5

DOI 10.3233/jid-2017-0016

http://www.sdpsnet.org

S D P S

\title{
Editorial
}

\section{Design and Evaluation of Integrated Healthcare Informatics}

\author{
Thomas T.H. Wan ${ }^{a}$, Varadraj P. Gurupur ${ }^{b *}$, and Murat M. Tanik ${ }^{c}$ \\ ${ }^{a}$ College of Health and Public Affairs, University of Central Florida, Orlando, FL, USA \\ ${ }^{b}$ Department of Health Management and Informatics, College of Health and Public Affairs, University of Central \\ Florida, Orlando, FL, USA \\ ${ }^{c}$ Department of Electrical and Computer Engineering, UAB School of Engineering, The University of Alabama at \\ Birmingham, Birmingham, AL, USA
}

Healthcare informatics research is a newly emerging interdisciplinary science to pursue the evidence-based information and knowledge applicable to enhance care management decisions and practices (Wan, 2006). The ultimate goal of this research is to guide administrative and clinical pursuits for excellence and to optimize health organizations' effectiveness and efficiency. Historically speaking, several scientific disciplines have established their research paradigms by employing a multidisciplinary perspective to improve health and health care. Their interdisciplinary approaches to personal and population health may include: 1) human ecology, a study of human adaptation and lifestyle in varying geospatial settings; 2) health demography, a study of vital events such as fertility, morbidity, mortality, and disability; 3) medical sociology, a study of synergism of social and environmental factors influencing health and illness of the population; 4) clinical epidemiology, a study of patterns and trends of morbidity and mortality associated with interventions and outcomes of care; 5) health economics, a study of consumption of health services, efficiency, and financial arrangements influencing the delivery of health care services; 6) health psychology, a study of behavioral-related factors such as attitude, perception, motivation, and preference for health related actions; 7) preventive medicine, a study of preventive strategies and interventions in the promotion of community and population health; 8) data science, in performing warehousing, mining, and statistical modeling of multilevel data to capture administrative and self-report data, to convert raw data into meaningful information, and to analyze data and formulate predictive analytics for health care improvement; and 9) health information science and management, a web of information networks enabling transformation and dissemination of systematic review and meta-analysis of results from clinical trial studies.

We have observed four emerging trends in healthcare informatics research around the globe (Wan, 2010; Kroneman et al, 2016; Jacobsen, 2008). First, healthcare researchers advocate the deployment of translational (converting basic science and knowledge into clinical practice for human health), transformational (directing a paradigm shift from a complex to parsimonious application of care management plans), and transdisciplinary investigations (integrating multi-factorial approaches into a coherent state of knowledge for enhancing behavioral and societal changes). Second, the technological innovations have driven the directions of knowledge management and development, information sharing and dissemination, and clinical care technology application. The availability of high-performance computers speeds up data processing and analytical capabilities of investigators. Third, collaborative opportunities among scientists and practitioners have shaped new research ventures in tackling common and special problems in the delivery of health care systems. There are excellent clinical programs

\footnotetext{
*Corresponding author. Email: varadraj.gurupur@ucf.edu.
} 
developed for caring patients with chronic diseases, such as the specialty accountable care organizations for oncology, heart disease and stroke care. These healthcare centers may operate under a progressive regulatory framework such as the value-based payment, patient-centered care and quality improvement programs. Fourth, the pursuit of casual inquiries in comparative effectiveness research for disease management and outcome assessment offers innovative solutions to handle population health management problems. The most notable example is to perform multilevel analysis of personal, organizational, ecological and contextual determinants of health (Wan et al., 2017). This approach has shed the light about the relevance of each contributing factor to the variation in healthcare outcomes. The role of confounders or extraneous variables can be delineated specifically so that the net influence of stronger personal and societal predictors can be identified. This is a helpful step to plan, implement, and evaluate intervention programs in population health management.

In the United States, as in other advanced countries, the five factors triggering health care management problems for chronic disease care are associated with the increase in the elderly population, the increased prevalence of multiple or poly chronic conditions, the need for containing costs of care, the need for improving efficiency and organizational performance, and the desire for patient-centric care (Wan, 2017).

The growth of aging population throughout the world is alarming and requires a thorough analysis of the demand for care, particularly for the increase of older old (75-80 years of age) and oldest old (85+). The conventional medical model for chronic care is too narrowed and very expensive if it is not adequately integrated with social care modalities such as community-based care and other alternative services.

Multiple or poly chronic conditions are often observed in the elderly population, irrespective of gender, race/ethnicity, and socioeconomic conditions of the people. Given the prevalence of poly conditions exists, little is known about the progression or trajectory changes of the disease process at the population level. Thus, health services researchers with the "big data" approach could explore the time-person-place trilogy of etiologies for poly chronic conditions, particularly in relationship to the investigation of metabolic related diseases (Murray et al., 2004; Wan, 2017).

Cost containment and related issues are complex problems that require a thorough investigation. The transition from the Affordable Care Act (the Obama Care) to the American Healthcare Act (the Trump Care) engenders serious concerns about the coverage of the uninsured and the preexisting conditions. Carefully designed value-based payment, incentive plan, and quality improvement programs in response to cost control and management problem are imperative.

The healthcare delivery systems are constantly under pressures to improve their efficiency and effectiveness (Wan, 1995; Grol et al., 2013). Health services management research plays a pivotal role in search for better designs and processes. It is unclear about the optimal relationship existed between the size-volume and quality of care. The United States is an innovative country that does not believe the use of one-size-fits-all strategy in the design of alternative healthcare organizations. More experimentations in the design and implementation of new healthcare organization are needed (Wan, 2002).

Patient-centered care such as Medical Homes is considered a popular solution to the primary care alternative in the United States (Rosenthal, 2008; Nielsen et al., 2016). The key principles in delivering primary and preventive care to the high-risk population include: 1) demand management ranging from needs assessment to patient engagement; 2) personalized care design (Hegarty et al., 2016); 3) use of health information technology to improve patient-physician communications and disease monitoring (Noblin, Wan and Fottler, 2013); 4) identification of mhealth utility; 5) encouragement or incentivization of preventive care practice and self-care management; and 6) promotion of community participation or engagement for the culture of health as noted in the Robert Wood Johnson Foundation's research initiative. It is interesting to note that the patient-centered care movement has fostered an emerging research discipline such as Personalized Care, particularly at the Southeastern University of Norway that offers a Ph.D. program for healthcare researchers.

The evolution of data science from the development of descriptive data analytics to predictive analytics has led to the detection of disease patterns and treatment plan variations for the chronic care population. However, the lack of specificities and conceptually grounded models prevents the formulation of effective predictive analytics that will help guide the policy interventions and changes needed. The fundamental questions in the system design applying to the relationships among the context, design, performance and outcome components of a healthcare system, are: 1) What are the mechanisms leading to better integrated chronic care models? 2) What are the causal paths for optimizing personal and population health outcomes in the implementation of an ideal system design? 3) For whom should the innovative care modality be targeted? 4) What are the uniform and minimal amounts of 
metrics required to assess clinical and self-reported outcomes in performance evaluation? 5) How can a theoretically informed and empirically validated framework be used in the design of decision support systems for improving organizational performance and patient care outcomes?

Disease management is a proactive approach to management of chronic conditions such as heart failure, hypertension, coronary heart disease, diabetes, COPD, asthma, and chronic kidney disorder through the provision of coordinated and integrated care to contain costs and improve patient care outcomes (Fiedler and Wan, 2010; Kroneman et al., 2016). A transdisciplinary approach to disease management is therefore proposed by integrating the macro- and micro-domains of a healthcare system. The macro system components include the contextual factors such as the socio-culture, political, and physical environmental aspects of the delivery system. The micro system components consist of personal-level and behavioral factors such as patients' knowledge $(\mathrm{K})$ about the disease and care process, motivation $(\mathrm{M})$ to change, attitude $(\mathrm{A})$ towards a specific treatment or care plan, and preventive care practice $(\mathrm{P})$. These KMAP components may either directly or indirectly affect the variability in patient care outcome measures. Disease management research should call for the integration of both micro- and macro-determinants of personal and population health (Wan, Terry, Cobb, McKee, and Kattan, 2017). Thus, the results can be used in the design and evaluation of decision support systems with the assistance of computer technologies and communication networks for improving self-efficacy and patient-centered care performance (Wan, Terry, Cobb, McKee, Tregerman and Barbaro, 2017).

Promoting a population health management strategy requires careful guidance from evidence-based research to shed some light on proof. Evidence is often accumulated from experiential and scientific knowledge through experimentation. One promising approach is to expand data mining efforts guided by a transdisciplinary research perspective coupled with the design of graphic-user interface (GUI)-based decision support systems (Wan, 2002). This enables researchers to validate and confirm the predictive analytics with large databases for multiple population groups. Ultimately, more efficient and effective care modalities, the evidence-based practice, can be developed from applying healthcare informatics research to optimize health and well-being of the population.

In this special issue we are including four articles in the aforementioned areas of health science. The article titled, "Healthcare - Probabilistic Techniques for Bone as a Natural Composite" discusses about probabilistic techniques used to model bone composites. This modeling takes into consideration uncertainties with regards to bone composites. In another article titled, “A 'Structured' Phenomenological Approach to Promoting Health among Young Adult and Adolescent Males" the authors establish the need to improve healthcare services. This need has been evaluated using "Young Adult and Adolescent Male (YAAM)" encounters. The experimentation described in this article establishes the need to improve proximal, intermediate, and distal health outcomes. "Mining Federated Data (MFD) - A Conceptual Framework for Exploration and Evaluation of Hospital Performance Measures” is an article that discusses about different methods used in evaluating the performance of a healthcare provider. Here the author provides a useful insight on the predictor variables used for this purpose. The author also provides information on the use of Enterprise Data Warehouse for achieving the same. Finally, the article titled "Case Study: Implementing and Integrating Health IT Solutions within a Correctional Environment” details the use of health information technology in prisons for inmates.

Society for Design and Process Science in particular promotes the importance of applying a scientific process to complex societal problems (Gurupur, et al., 2016; Gurupur and Gutierrez, 2016). Generally speaking, these problems are transdisciplinary in nature; therefore, solutions driven from convergence of research have to be taken into account to address these complex problems (Martis, et al., 2017; NSF, 2017; Gurupur and Wan, 2017). It is our opinion that Healthcare Informatics is one such field and we have hereby attempted to address some of the complexities of this field in this special issue.

\section{References}

Fiedler, B.A., and Wan, T.T.H. (2010). Disease management organization approach to chronic illness. International Journal of Public Policy 6(3/4): 260-277. 
Gurupur, V., Wan, T.T.H., Malvey, D., Slovensky, D., (2016). Editorial: Design of Health Information Systems, Journal of Integrated Design and Process Science, Vol. 20 (1), pp. 3-6.

Gurupur, V., Wan, T.T.H., (2017). Current state and challenges in implementing mHealth: A technical perspective, mHealth, DOI: 10.21037/mhealth.2017.07.05.

Gurupur, V., Gutierrez, R., (2016). Designing the Right Framework for Healthcare Decision Support, Journal of Integrated Design and Process Science, DOI: 10.3233/jid-2016-0001.

Grol, R., Wensing, M., Eccleg, M. and Davis, D. (2013). Improving Patient Care: The Implementation of Change in Health Care ( $2^{\text {nd }}$ edition). New York: the Wiley-Blackwell.

Hegarty, C., Buckley,C., Forrest, R., and Marshall, B. (2016). Discharge planning: Screening older patients for multidisciplinary team referral. International Journal of Integrated Care 16(4):1. doi.10.5334/ijic.22252.

Jacobsen, K.H. (2008). Introduction to Global Health. Sudbury, MA: Jones and Bartlett Publishers.

Kroneman, M., Boerma, W., vanden Berug, M., Groenewegen, P., de yong, J., and van Ginneken, E. (2016). Health system in transition. The Netherlands Health System Review 18(2): 1-239.

Martis, R. J., Lin, H., Gurupur, V., Fernandes, S.L., (2017). Editorial: Frontiers in Development of Intelligent Applications for Medical Imaging Processing and Computer Vision, Computers in Biology and Medicine, DOI:10.1016/j.compbiomed.2017.06.008.

Murray, C.J., Lopez, A.D. and Wibulpolprasert, S. (2004). Monitoring global health: time for new solutions. British Medical Journal 329:1096-1100.

NSF Online: https://www.nsf.gov/od/oia/convergence/index.jsp. Last Accessed: 10/23/2017.

Nielsen, M., Buelt, L., Patel, K. and Nichols, L.M. (2016). The Patient-Centered Medical Home's Impact on Cost and Quality: Annual Review of Evidence, 2014-2015. Patient-Centered Primary Care Collaborative.

https://www.pcpcc.org/sites/default/files/resources.

Noblin, A., Wan, T.T.H., and Fottler, M. (2013). Intention to use a personal health record: a theoretical analysis using the technology acceptance model. International Journal of Healthcare Technology and Management, 14(1/2): 73-89.

Rosenthal, T.C. (2008). The medical homes: Growing evidence to support a new approach to primary care. Journal of American Board of Family Medicine 21: 427-440.

Wan, T.T.H. (1995). Analysis and Evaluation of Health Care Systems: An Integrated Approach to Managerial Decision Making. Baltimore: Health Professions Press.

Wan, T.T.H. (2002). Evidence-Based Health Care Management: Multivariate Modeling Approaches. Boston: Kluwer Academic Publishers.

Wan, T.T.H. (2006). Healthcare informatics research: from data to evidence-based practice. Journal of Medical Systems 30 (1): 3-7.

Wan, T.T.H. (2010). Global health research strategies. International Journal of Public Policy 5(2/3): 104-120.

Wan, T.T.H., Terry, A., McKee, B., and Kattan, W. (2017). A KMAP-O framework for care management research of patients with type 2 diabetes. The World Journal of Diabetes 8(4): 165-171. DOI: 10.4239/wjd.v8.i4.165. 
Wan, T.T.H., Terry, A., Cobb, E., McKee, B., Tregerman, R., and Barbaro, S.D.S. (2017). Strategies to modify the risk of heart failure readmission: A systematic review and meta analysis. Health Services Research-Managerial Epidemiology 4: 1-16. 